\title{
Interstitial pneumonia associated with autoimmune diseases: a possible mimicker of SARS-CoV-2 pneumonia
}

\author{
Maria Rosa Pozzi ${ }^{1}$ Paola Faverio ${ }^{3,4}$. Valentina Varisco ${ }^{1}$ - Gabriele D'Andrea ${ }^{2}$. Angela Concetta Giuffrida ${ }^{5}$. \\ Fabrizio Luppi ${ }^{3,4}$
}

Received: 8 May 2021 / Accepted: 7 September 2021 / Published online: 12 October 2021

(c) Società Italiana di Medicina Interna (SIMI) 2021

Dear Sir,

The COVID-19 pandemic, caused by SARS-CoV-2, has expanded dramatically throughout the world [1]. The majority of patients with COVID-19 has a good prognosis, but there is a significant proportion of patients requiring intensive care [1].

Acute interstitial pneumonia and acute respiratory distress syndrome (ARDS) are the hallmark of severe COVID19 [1]. In these patients, it has been hypothesized that an abnormal immunological response, with features partially overlapping with hemophagocytic lymphohistiocytosis (HLH) or cytokine storm syndrome (CSS) may play a crucial role [2].

The SARS-COV-2 pneumonia is characterised by peripheral bilateral ground glass opacities (GGO) with or without consolidation. Multifocal GGO of round morphology and features of organizing pneumonia are also reported [3].

The pulmonary involvement in autoimmune diseases (CTD-ILD) ranges from mild to rapidly progressive respiratory failure and ARDS [4]. Adult onset Still disease (AOSD) is mainly characterized by systemic inflammation and often is associated with HLH [5]. Although pulmonary involvement occurs only in $5 \%$ of the patients, ARDS can be observed at disease onset, usually in patients with a severe systemic pattern [6].

Maria Rosa Pozzi

m.pozzi@asst-monza.it

1 Rheumatology Unit, S. Gerardo Hospital, Via Pergolesi, 33, 20900 Monza, Italy

2 Radiology Unit, S. Gerardo Hospital, Monza, Italy

3 Pneumology Unit, S. Gerardo Hospital, Monza, Italy

4 Department of Medicine and Surgery, University of Milano Bicocca, Milan, Italy

5 Intensive Care Unit, San Gerardo Hospital, Monza, Italy
Beyond clinical and radiological features, SARS-CoV-2 pneumonia and CTD-ILD share prognostic factors such as lymphopenia, increased neutrophil-to-lymphocyte ratio (N/L ratio), hyperferritinemia [7-9]. High D-dimer levels are associated with a poorer outcome in COVID-19 [10]. Similarly, D-dimer elevation is linked with the risk of developing acute exacerbation in CTD-ILD [10].

We describe three cases of patients hospitalized between February and April 2020 initially misdiagnosed with COVID-19 but that were ultimately affected by CTD-ILD.

Case 1. Forty-four year-old female suffering of arthralgias, low grade fever and hyporexia since March 2020. Blood tests showed mild anemia, neutrophilic leukocytosis, c-reactive protein (CRP) elevation; rheumatoid factor (RF) and anti-nuclear antibody (ANA) resulted negative. She was admitted for worsening of arthralgias with symmetric polyarthritis of small joints and knees, fever and odynophagia. Chest X-ray showed a small consolidation in right lower lobe and mild opacities in right upper lobe and hypoxemia (partial pressure of oxygen in the arterial blood $-\mathrm{PaO}_{2}-54 \mathrm{~mm}$ $\mathrm{Hg}$ ) was detected. SARS-CoV-2 RNA resulted negative in two naso-pharyngeal swabs. Leukocytosis was observed $\left(18.6 / 10^{3} / \mathrm{uL}\right)$ with neutrophilia, lymphopenia and neutrophil/lymphocyte (N/L) ratio 17.14. High levels of CRP, anti-citrullinated protein antibodies (ACPA) and Anti-Ro52 antibodies were observed. Blood and urine cultures, urinary Pneumococcus and Legionella Pn. antigens were negative (Table 1).

Low-dose steroid and hydroxychloroquine to treat arthritis and ceftriaxone plus azithromycin for pneumonia were administered. After a few days, she developed high fever, dyspnea, cough and severe desaturation $\left(\mathrm{PaO}_{2} 40 \mathrm{~mm} \mathrm{Hg}\right)$. High-resolution computed tomography (HRCT) revealed multiple peripheral GGO, predominantly in the lower lung and limited fibrosis (Fig. 1a); SARS-CoV-2 as well as extensive microbiological testing on bronchoalveolar lavage (BAL) were all negative (Table 1): Anti-SARS-CoV-2 
Table 1 Laboratory and radiological features

\begin{tabular}{|c|c|c|c|c|c|c|c|c|}
\hline & \multicolumn{3}{|c|}{ Case 1 (SC, F, 44 years) } & \multicolumn{2}{|c|}{ Case 2 (AM, F, 71 years) } & \multicolumn{3}{|c|}{ Case 3 (VM, M, 53 years) } \\
\hline & Baseline & ICU admission & Discharge & Baseline & Discharge & Baseline & Myositis & Last follow-up \\
\hline $\mathrm{CRP}(\mathrm{mg} / \mathrm{dl})$ & 10.37 & 16.17 & 1.1 & 17.09 & 0.66 & 3.15 & 3.18 & 0.15 \\
\hline $\begin{array}{l}\text { Procalcitonin } \\
\quad(<0.5 \mathrm{ng} / \mathrm{ml})\end{array}$ & 2.71 & 0.15 & 0.06 & 0.6 & 0.14 & 0.08 & nd & $\mathrm{Nd}$ \\
\hline $\begin{array}{l}\mathrm{ANC}\left(10^{3} / \mathrm{uL}\right. \\
\text { range } 1.5-7.5)\end{array}$ & 16.12 & 23.81 & 6 & 8.64 & 4.83 & 6.67 & 9.64 & 2.8 \\
\hline $\begin{array}{l}\text { Lymph }\left(10^{3} / \mathrm{uL}\right. \\
\text { range } 1-5)\end{array}$ & 0.94 & 0.36 & 2.41 & 1.34 & 1.08 & 1.94 & 1.64 & 2.66 \\
\hline $\begin{array}{l}\text { NLR (range } \\
1.65-3.53 \text { )* }\end{array}$ & 17.14 & 66.13 & 2.49 & 6.4 & 4.47 & 3.43 & 5.87 & 1.05 \\
\hline Platelets $\left(10^{3} / \mathrm{uL}\right)$ & 514 & 319 & 418 & 338 & 171 & 360 & 499 & 336 \\
\hline $\begin{array}{l}\text { Ferritin (ng/dl rang } \\
13-150)\end{array}$ & 118 & 195 & 98 & 511 & nd & 414 & nd & 235 \\
\hline $\begin{array}{l}\mathrm{D} \text {-dimer }(\mathrm{ng} / \mathrm{ml} \\
\text { range } 0-250)\end{array}$ & 786 & 2640 & 468 & 353 & nd & 942 & 680 & $\mathrm{Nd}$ \\
\hline $\begin{array}{l}\text { Haemoglobin }(\mathrm{g} / \mathrm{dl} \\
\text { range } 12-16)\end{array}$ & 10.7 & 9.4 & 10.9 & 12.6 & 9.9 & 13.6 & 13.6 & 14.6 \\
\hline $\begin{array}{l}\mathrm{CK}(\mathrm{U} / \mathrm{L} \\
\quad \text { range }<170)\end{array}$ & 33 & 50 & 100 & 66 & 13 & 201 & 1524 & 42 \\
\hline $\begin{array}{l}\text { LDH (U/L range } \\
135-214)\end{array}$ & 367 & 431 & nd & 206 & nd & 244 & 371 & nd \\
\hline AST $(\mathrm{U} / \mathrm{L}<32)$ & 8 & 26 & 18 & 22 & 45 & 22 & 52 & 10 \\
\hline ALT $(\mathrm{U} / \mathrm{L}<33)$ & 22 & 40 & 15 & 9 & 28 & 28 & 44 & 11 \\
\hline $\begin{array}{l}\text { Tryglicerides }(\mathrm{mg} / \\
\mathrm{dl}<150)\end{array}$ & 75 & 205 & 96 & $\mathrm{Nd}$ & nd & nd & nd & nd \\
\hline $\mathrm{pO} 2(\mathrm{mmHg})$ & 54 & 40.6 (LF O2) & nd & 45.4 (AA) & $60(\mathrm{AA})$ & 57(AA) & nd & nd \\
\hline $\mathrm{pCO} 2(\mathrm{mmHg})$ & 41 & 31.1 & & 27.8 & 37.5 & 35.3 & nd & nd \\
\hline $\mathrm{O} 2$ saturation $\%$ & 88 (AA) & 78 (LF O2) & 96 (AA) & 86 (AA) & $93(\mathrm{AA})$ & $92(\mathrm{AA})$ & 94 (AA) & 97 (AA) \\
\hline $\begin{array}{l}\text { SARS-CoV-2 RNA } \\
\text { (nasopharyng. } \\
\text { swab) }\end{array}$ & Neg (2 samples) & & & $\mathrm{Neg}$ & & Neg (4 samples) & nd & \\
\hline $\begin{array}{l}\text { SARS-CoV-2 RNA } \\
\text { (BAL) }\end{array}$ & & Neg & & Neg & & nd & & \\
\hline $\begin{array}{l}\text { Anti-SARS-CoV-2 } \\
\text { antibodies }\end{array}$ & & Neg & & & & & Neg & \\
\hline $\begin{array}{l}\text { Haemocoltures } \\
\text { (aerob./anaerob) }\end{array}$ & $\mathrm{Neg}$ & & & $\mathrm{Neg}$ & & nd & & \\
\hline $\begin{array}{l}\text { Urinary Antigens } \\
\text { Legionel./Pneum }\end{array}$ & $\mathrm{Neg}$ & & & $\mathrm{Neg}$ & & $\mathrm{Neg}$ & & \\
\hline $\mathrm{BAL}^{\&}$ & & Neg & & $\mathrm{Neg}$ & & nd & & \\
\hline ANA & Neg & & & Pos, 1.640, & ere & $\mathrm{Neg}$ & & \\
\hline Rheumatoid factor & Neg & & & nd & & Neg & & \\
\hline Other auto Abs & $\operatorname{Ro5} 2(2+)$, ACPA & $\mathrm{A}(1550 \mathrm{U} / \mathrm{ml})$ & & $\operatorname{Ro52}(3+)$ & $\mathrm{M} 2, \mathrm{EJ}(2+)$ & PL7 (3+) & & \\
\hline HTRC GGO & ++ & & & ++ & & ++ & & \\
\hline HRTC reticulation & + & & & + & & - & & \\
\hline $\begin{array}{l}\text { HRTC consolida- } \\
\text { tion }\end{array}$ & + & & & ++ & & + & & \\
\hline
\end{tabular}

*See reference [13]

${ }^{\&}$ BAL (Bronchoalveolar lavage): Multiplex Respiratory RNA/DNA virus: Influenza A- H1, H1N1, H3, Influenza B, RSV A,B, Adenovirus, human Metapneumovirus, Coronavirus 229E, NL63,OC43, Parinfluenza virus 1/2/3/4, Rinovirus A/B/C, Human Entrovirus, Human Bicavirus1/2/3/4. Muliplex Respiratory DNA bacteria: Mycoplasma pneumoniae, Legionella pneumophila, Bordetella parapertussis, Haemophilus Influenzae, Bordetella pertussis, Chlamydophila pneumoniae, Streptococcus pneumoniae, Mycobacterium TB-complex Bacterioscopic: Acidfast stain Gram stain. Pneumocystis jerovecii, galactomannan Ag Coltural: aerobic bacteria and fungi, mycobacteria 


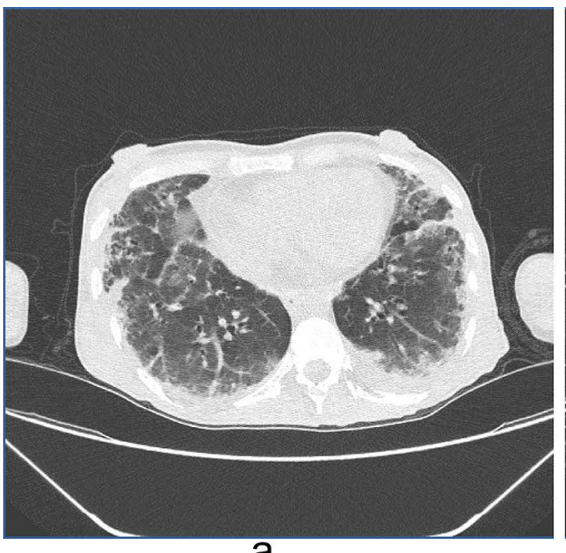

a

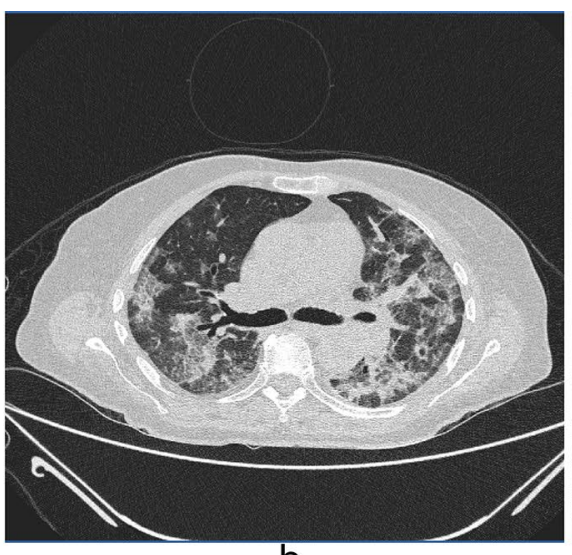

b

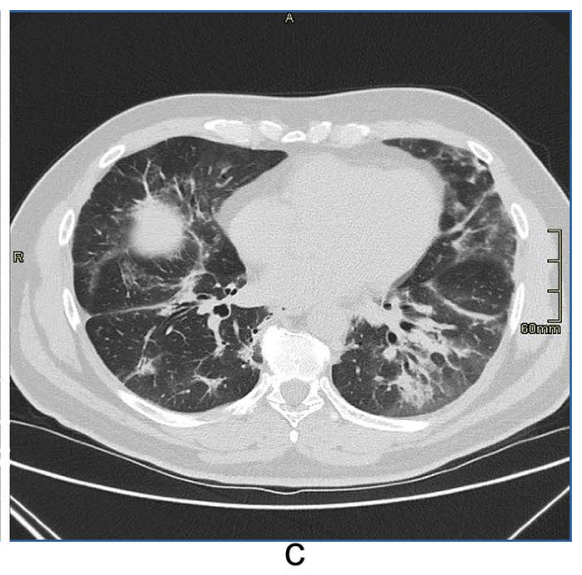

Fig. 1 a Lower lung scan showing ground-glass-opacities (GGO) with mild reticulation in a peripheral, lower lung zone distribution. Minimal traction bronchiolectasis is present in middle lobe. b Upper lung scan showing ground-glass-opacities (GGO) with subpleural sparing, consolidations with perilobular distribution in keeping with

IgG were in the normal range. White blood cell (WBC) count with $\mathrm{N} / \mathrm{L}$ ratio 66.13 , CRP, ferritin, D-dimer, triglycerides and lactate deydrogenase (LDH), but not procalcitonin, increased, whereas haemoglobin and platelets count decreased (Table 1). Despite aggressive antibiotic therapy, acute respiratory failure worsened. The patient underwent to endotracheal intubation (ETI) for the occurrence of ARDS and moved to Intensive Care Unit (ICU), where pronation protocol was applied: unfortunately, the respiratory failure did not improve. We concluded that ARDS was a manifestation of AOSD, because of the presence of arthritis along with neutrophilic leukocytosis, high fever, odynophagia and biomarkers consistent with this diagnosis, even though some typical clinical manifestations, such as maculopapular rash, lymphoadenopathy, hepatosplenomegaly, or serositis were missing in our patient and ferritin levels were just above upper normal limit. Nevertheless, although Yamaguchi's criteria suggest that ANA and RF should be negative, a few data support anti-CCP and antiSSA/Ro52 antibodies positivity in AOSD. Therefore, we concluded for an atypical form of AOSD diagnosis.

We treated patient with pulse corticosteroid therapy (methylprednisolone, $1000 \mathrm{mg} /$ day) for three days. We did not observe any radiological and functional improvement after the three pulses and, therefore, we introduced iv anakinra (r-metHuIL-1ra), $100 \mathrm{mg}$ QID for 15 days, with a dramatic improvement of the clinical picture, leading to extubation and progressive reduction of oxygen therapy. Prednisone-equivalent $1 \mathrm{mg} / \mathrm{kg} /$ day for 2 weeks and subsequent taper of $25 \%$ of the daily dose every 2 weeks till the dose of $12.5 \mathrm{mg} /$ day was administered. The patient was discharged with low activity arthritis, no cough or dyspnea, normal oxygen saturation and normal blood cell count, CRP organizing-pneumonia. c Lower lung scan showing ground-glassopacities (GGO) and consolidations with peribronchovascular and perilobular distribution in keeping with mixed pattern of NSIP and organizing-pneumonia

and ferritin, still on low-dose prednisone, hydroxychloroquine and anakinra $100 \mathrm{mg} /$ day/sc. The HRCT performed after 5 months showed resolution of consolidations, but subpleural reticulation and some traction bronchiectasis persisted.

Case 2 . Seventy-one-year-old female affected by primary biliary cholangitis, diagnosed in 2006 on clinical grounds (persistent increase of cholestatic enzymes and IgM plasmatic level, ANA and anti-mitochondrial M2 autoantibodies positivity) even though liver histology was inconclusive, sicca syndrome and Raynaud's phenomenon without cutaneous features of SSc. Since then anticentromere antibodies (ACA) were present, but not anti SSA/Ro52. In February 2020, she was treated with amoxicillin-clavulanate for cough, dyspnea and fever. She was admitted with respiratory failure $\left(\mathrm{PaO}_{2} 45 \mathrm{~mm} \mathrm{Hg}\right)$, fever and bilateral pneumonia. Blood tests revealed mild leukocytosis with $\mathrm{N} / \mathrm{L}$ ratio of 6.4, increased CRP and ferritin. SARS-CoV-2 RNA was negative both on nasopharyngeal and bronchoalveolar lavage (BAL) samples. Blood cultures, BAL microbiological testing and urinary antigens for Legionella Pn. and Pneumococcus resulted negative (Table 1). Continuous positive airway pressure (CPAP) and pronation protocols were applied; ceftriaxone and azithromycin followed by piperacillin-tazobactam, levofloxacin and ceftaroline fosamil were administered empirically, with radiological worsening. Chest HRCT showed bilateral GGO with subpleural sparing, perilobular consolidations in the upper lung with extensive consolidations and fibrosis in lower lobes (Fig. 1b). High titer ANA with centromere pattern, anti-mitochondrial antibody (AMA)-M2, Anti-Ro52 and EJ autoantibodies resulted positive. Therefore, an anti-synthetase syndrome (ASS) was diagnosed and methylprednisolone $(1 \mathrm{mg} / \mathrm{kg} /$ day $)$ and 
mycophenolate mofetil (MMF) were started with clinical and radiological improvement. The patient was discharged without respiratory failure; CRP and WBC count were normal (Table 1). After 7 months chest HRCT showed complete regression of consolidations, with persistence of subpleural reticulation at lower lobes and a few traction bronchiectasis.

Case 3. Fifty-three-year-old male, complained about exertional dyspnea, cough and low-grade fever in February 2020, seeking medical attention for progressive dyspnea. He underwent immediately to CPAP for severe respiratory failure $\left(\mathrm{PaO}_{2} 57 \mathrm{~mm} \mathrm{Hg}, \mathrm{O}_{2} \mathrm{Sat} 92 \%\right)$. Two nasopharyngeal swabs for SARS-CoV-2 resulted negative. Increased CRP, D-dimer, ferritin and creatin phosphokinase (CK) levels were detected (Table 1). HRCT showed bilateral multiple patchy areas of GGO and consolidations with peribronchovascular and perilobular distribution at lower lobes (Fig. 1c).

Despite undetectable SARS-CoV-2 RNA, based on suspicion of COVID-19 pneumonia, he was treated with lopinavir-ritonavir, hydroxychloroquine, azithromycin, ceftriaxone and prophylactic low-molecular-weight heparin (LMWH). Two further nasopharyngeal swabs for SARS-CoV-2 resulted negative. Respiratory failure improved and the patient was moved to a rehabilitation hospital with low-flow oxygen. At the end of April, he was discharged, although persistent exertional dyspnea, fever and weight loss, worsening fatigue, myalgias and arthralgias. An HRCT in July 2020 showed improvement of both GGO and consolidation areas. Pulmonary function tests (PFT) showed a restrictive pattern with a forced vital capacity (FVC) $51 \%$ of the predicted values, carbon monoxide diffusion capacity (DLCO) 43\%, of the predicted values and $\mathrm{O} 2$ saturation was $94 \%$ in room air. Blood tests showed a significant increase of creatin phosphokinase (CK) (1500 U/L) and high positivity for anti PL-7 antibody (Table 1). ASS diagnosis was made and prednisone plus mycophenolate mofetil (MMF) were started, with a rapid improvement of both systemic and respiratory symptoms. After 2 months CK, CRP and ferritin levels resulted normal, the patient markedly recovered, complaining only of a mild exertional dyspnea and fatigue. At that time, PFT resulted normal (FVC 100\% and DLCO 74\% of the predicted values) and $\mathrm{O}_{2}$ saturation was $96 \%$ in room air.

\section{Discussion}

We described a case series of three patients affected by CTD-ILDs showing clinical and radiological overlapping features with SARS-CoV-2 pneumonia during the COVID-19 pandemic. COVID-19 pneumonia can evolve to ARDS, the leading cause of mortality. The marked elevation of pro-inflammatory cytokines and chemokines, hyperferritinemia and coagulopathy suggests that excessive immune responses associated with HLH/CSS may be driving COVID-19-related ARDS. It is still unclear if HLH in COVID-19 is due to exaggerated cytokine response triggered by an impaired antiviral defence, with type-I interferon suppression [11] or if an hypersensitive T-cell-mediated reaction against viral infected cells contributes to lung damage [2]. This immunological response in COVID-19 patients appears to be confined to the lung, without multisystem involvement typical of HLH associated with infections or autoimmune diseases [12]. AOSD is frequently complicated by HLH, although the ARDS is uncommon [6]. In our cases, the lung damage was the only visceral manifestation, beside the arthritis, and this made the diagnosis more challenging during the pandemic.

The high-dose steroid was insufficient to overcome respiratory failure and IL-1 blocking therapy was crucial to obtain a marked clinical improvement, including tracheal extubation.

The radiological picture of SARS-CoV-2 pneumonia shares some features with ASS lung involvement. Typical CT findings in ASS-ILD are considered areas of groundglass attenuation with peripheral and peribronchovascular distribution (non-specific interstitial pneumonia pattern), often associated with consolidations [13], sometimes with progression towards fibrosis. COVID-19 pneumonia is usually characterised by bilateral GGO opacities, but organizing pneumonia and fibrosis are also reported [3].

ASS belongs to a heterogeneous group of Idiopathic Inflammatory Myopathies; the main manifestations of ASS are myositis, ILD, fever, Raynaud phenomenon and mechanic's hands. ILD is the first manifestation in 54-74\% of PL-7/EJ ASS cases and the isolated involvement in 34-39\% of patients [13]. In cases 2 and 3, lung involvement was the only manifestation at onset. The ongoing pandemic increased acceptance of an incorrect diagnosis of COVID-19 pneumonia and delayed the diagnosis of auto-immune pneumonia and the appropriate treatment, with a potential worsening in prognosis.

In conclusion, we reported three cases highlighting clinical and radiological similarities between COVID-19 pneumonia and lung involvement in CTD-ILD characterized by acute progression and signs of innate immunity activation. Interestingly Ro52/TRIM21, that binds intracellular antibody-bound pathogens including RNA viruses [14] and melanoma differentiation-associated protein (MDA)-5, a sensor of viral RNA [15], are the antigenic targets of autoantibodies which are markers of lung involvement in CTD.

Our study highlights the importance of an appropriate differential diagnosis between CTD-ILD and SARS-CoV-2 pneumonia, even in the course of COVID-19, in consideration of a different management and of the need of a different hospital accommodation. 
Author contributions PF, VV, and AG collected clinical cases, MRP and FL wrote the manuscript. GDA analyzed chest computed tomographies. All authors read and approved the final manuscript.

Funding This research did not receive any specific grant from funding agencies in the public, commercial, or not-for-profit sectors.

Availability of data and material Not applicable.

Code availability Not applicable.

\section{Declarations}

Conflict of interest FL and PF report personal fee from Roche and Boehringer-Ingheleim, outside the submitted work. All the other authors have no conflict of interest to declare.

Ethics approval Not applicable.

Informed consent Patients provided written informed consent to be part of this study.

Consent for publication Patients provided written informed consent for publication.

\section{References}

1. Guan W-J, Ni Z-Y, Hu Y et al (2020) Clinical characteristics of coronavirus disease 2019 in China. N Engl J Med 382:1708-1720. https://doi.org/10.1056/NEJMoa2002032

2. McGonagle D, Sharif K, O'Regan A, Bridgewood C (2020) The role of cytokines including interleukin-6 in COVID-19 induced pneumonia and macrophage activation syndrome-like disease. Autoimmun Rev 19:102537. https://doi.org/10.1016/j.autrev.2020. 102537

3. Simpson S, Kay FU, Abbara S et al (2020) Radiological society of north america expert consensus statement on reporting chest CT findings related to COVID-19. Endorsed by the Society of Thoracic Radiology, the American College of Radiology, and RSNA - secondary publication. J Thorac Imaging 35:219-227. https:// doi.org/10.1097/RTI.0000000000000524

4. Paschalaki KE, Jacob J, Wells AU (2016) Monitoring of lung involvement in rheumatologic disease. Respiration 91:89-98. https://doi.org/10.1159/000442890

5. Bae C-B, Jung J-Y, Kim H-A, Suh C-H (2015) Reactive hemophagocytic syndrome in adult-onset still disease. Medicine
(Baltimore) 94:e451. https://doi.org/10.1097/MD.0000000000 000451

6. Gerfaud-Valentin M, Cottin V, Jamilloux Y et al (2016) Parenchymal lung involvement in adult-onset Still disease: a STROBEcompliant case series and literature review. Medicine (Baltimore) 95:e4258. https://doi.org/10.1097/MD.0000000000004258

7. Henry BM, de Oliveira MHS, Benoit S et al (2020) Hematologic, biochemical and immune biomarker abnormalities associated with severe illness and mortality in coronavirus disease 2019 (COVID19): a meta-analysis. Clin Chem Lab Med 58:1021-1028. https:// doi.org/10.1515/cclm-2020-0369

8. Shi J, Li S, Yang H et al (2017) Clinical profiles and prognosis of patients with distinct antisynthetase autoantibodies. J Rheumatol 44:1051-1057. https://doi.org/10.3899/jrheum.161480

9. Ha Y-J, Hur J, Go DJ et al (2018) Baseline peripheral blood neutrophil-to-lymphocyte ratio could predict survival in patients with adult polymyositis and dermatomyositis: a retrospective observational study. PLOS ONE 13:e0190411. https://doi.org/10.1371/ journal.pone.0190411

10. Ishikawa G, Acquah SO, Salvatore M, Padilla ML (2017) Elevated serum D-dimer level is associated with an increased risk of acute exacerbation in interstitial lung disease. Respir Med 128:78-84. https://doi.org/10.1016/j.rmed.2017.05.009

11. Soy M, Keser G, Atagündüz P et al (2020) Cytokine storm in COVID-19: pathogenesis and overview of anti-inflammatory agents used in treatment. Clin Rheumatol 39:2085-2094. https:// doi.org/10.1007/s10067-020-05190-5

12. McGonagle D, O'Donnell JS, Sharif K et al (2020) Immune mechanisms of pulmonary intravascular coagulopathy in COVID-19 pneumonia. Lancet Rheumatol 2:e437-e445. https://doi.org/10. 1016/S2665-9913(20)30121-1

13. Cavagna T-A et al (2019) Influence of antisynthetase antibodies specificities on antisynthetase syndrome clinical spectrum time course. J Clin Med 8:2013. https://doi.org/10.3390/jcm8112013

14. Xue B, Li H, Guo M et al (2018) TRIM21 promotes innate immune response to RNA viral infection through Lys27-linked polyubiquitination of MAVS. J Virol. https://doi.org/10.1128/JVI. 00321-18

15. Dias Junior AG, Sampaio NG, Rehwinkel J (2019) A balancing Act: MDA5 in antiviral immunity and autoinflammation. Trends Microbiol 27:75-85. https://doi.org/10.1016/j.tim.2018.08.007

Publisher's Note Springer Nature remains neutral with regard to jurisdictional claims in published maps and institutional affiliations. 